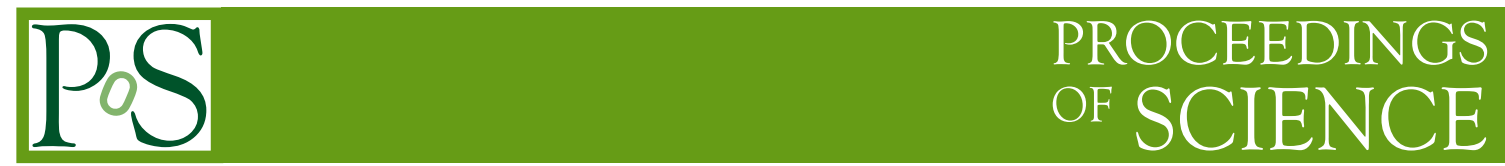

\title{
Inert Model and evolution of the Universe
}

\author{
Dorota Sokołowska*† \\ University of Warsaw \\ E-mail: dsokefuw.edu.pl
}

We consider evolution of the Universe after EWSB leading to the present inert phase, containing a SM-like Higgs boson and scalar dark particles, among them a Dark Matter candidate. We address the question, if there is a possibility to have a sequence of the phase transitions instead of a single one leading directly from EW symmetric phase to the inert one.

35th International Conference of High Energy Physics

July 22-28, 2010

Paris, France

*Speaker.

${ }^{\dagger}$ Work done in collaboration with I.F. Ginzburg, K.A. Kanishev (Novosibirsk University) and M. Krawczyk (University of Warsaw). Work was partly supported by Polish Ministry of Science and Higher Education Grant N N202 230337, FLAVIAnet contract No.MRTN-CT-2006-035482 and EC 6th Framework Programme MRTN-CT-2006-035863. 
We consider the 2HDM Lagrangian for two $S U(2)$ scalar doublets $\varphi_{S}, \varphi_{D}$ with Yukawa interaction set to Model I and the potential $V$ :

$V=-\frac{m_{11}^{2}}{2}\left|\varphi_{S}\right|^{2}-\frac{m_{22}^{2}}{2}\left|\varphi_{D}\right|^{2}+\frac{\lambda_{1}}{2}\left|\varphi_{S}\right|^{4}+\frac{\lambda_{2}}{2}\left|\varphi_{D}\right|^{4}+\lambda_{3}\left|\varphi_{S}\right|^{2}\left|\varphi_{D}\right|^{2}+\lambda_{4}\left|\varphi_{S}^{\dagger} \varphi_{D}\right|^{2}+\frac{1}{2}\left(\lambda_{5}\left(\varphi_{S}^{\dagger} \varphi_{D}\right)^{2}+h . c\right)$.

$V$ is invariant under a $Z_{2}$ transformation: $\varphi_{S} \rightarrow \varphi_{S}, \varphi_{D} \rightarrow-\varphi_{D}$. In the Inert Model also vacuum state is $Z_{2}$-symmetric and the Dark Matter candidate, from the $Z_{2}$-odd doublet $\varphi_{D}$, appears.

The most general EWSB solution $\left\langle\varphi_{S}\right\rangle^{T}=\frac{1}{\sqrt{2}}\left(0, v_{S}\right),\left\langle\varphi_{D}\right\rangle^{T}=\frac{1}{\sqrt{2}}\left(u, v_{D}\right)$ gives three neutral extrema $(u=0)$ : inert $\left(I_{1} ; v_{D}=0, v_{S}^{2}=v^{2}\right)$ with SM-like Higgs $h$ from $\varphi_{S}$ and DM candidate from $\varphi_{D}$ (eg. $H$ ); inert-like $\left(I_{2} ; v_{S}=0, v_{D}^{2}=v^{2}\right)$ with massless fermions (Model I: only $\varphi_{S}$ couples to fermions) and no candidate for DM; mixed $\left(M ; v_{D}, v_{S} \neq 0, v^{2}=v_{S}^{2}+v_{D}^{2}\right)-$ a standard 2HDM extremum. The lowest energy extremum, fulfilling positivity constraints, is the vacuum.

The one-loop thermal corrections to $V$ are $m_{i i}^{2}(T)=m_{i i}^{2}-c_{i} T^{2}(i=1,2)$ where $c_{i}=c_{i}\left(\lambda_{1-4} ; g, g^{\prime}\right.$; $g_{t}^{2}+g_{b}^{2}$ for $\left.i=1\right)$, with fixed $\lambda_{i}\left(g, g^{\prime}-\right.$ EW gauge couplings, $g_{t}, g_{b}-$ SM Yukawa couplings).

The possible sequences of phase transitions between different vacua are shown as rays in plots (1a-1c), for $\left(\mu_{1}, \mu_{2}\right)$ plane $\left(\mu_{i}(T)=m_{i i}^{2}(T) / \sqrt{\lambda_{i}}, i=1,2\right)$. For $E W \rightarrow I_{1}, I_{1}$ is the only vacuum that existed after EWSB. For rays I, VI, IX $I_{2}$ is not an extremum, for rays II, VII $I_{2}$ is an extremum, but not a local minimum; for ray III $I_{2}$ is a local minimum. The sequence: $E W \rightarrow I_{2} \rightarrow I_{1}$, with transition between $I_{2}$ and $I_{1}$ vacuum, is possible for ray $\mathbf{I V}$, where $I_{2}$ is not a local minimum and for ray $\mathbf{V}$, where $I_{2}$ is a local minimum, that coexists with the global minimum $I_{1}$. For ray VIII there is a possibility of going through the mixed vacuum $E W \rightarrow I_{2} \rightarrow M \rightarrow I_{1}$. In this case there is only one minimum at any temperature.

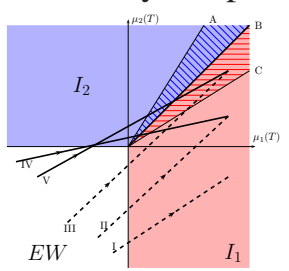

(a) $\lambda_{345}>\sqrt{\lambda_{1} \lambda_{2}}$

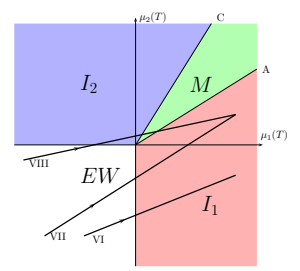

(b) $0<\lambda_{345}<\sqrt{\lambda_{1} \lambda_{2}}$

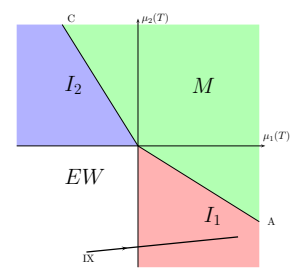

(c) $-\sqrt{\lambda_{1} \lambda_{2}}<\lambda_{345}<0$

Figure 1: Possible rays from EW symmetric phase to the inert phase $I_{1} ; \lambda_{345}=\lambda_{3}+\lambda_{4}+\lambda_{5}$.

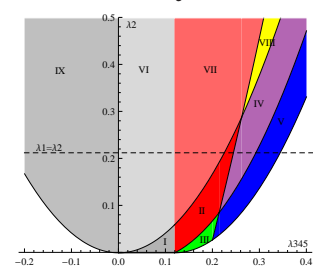

(a) with fermions

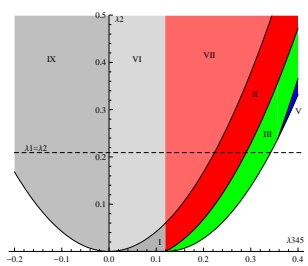

(b) without fermions

Figure 2: Example of rays for $M_{h}=120 \mathrm{GeV}, M_{H}=60 \mathrm{GeV}, M_{A}=68 \mathrm{GeV}, M_{H^{ \pm}}=110 \mathrm{GeV}$.

Plots 2a,2b show the different vacua sequences (rays I-IX) leading to $I_{1}$ in $\left(\lambda_{2}, \lambda_{345}\right)$ plane. For $\lambda_{2} \leq \lambda_{1}$ different types of vacua in the past are possible only if the fermionic part of $c_{1}$ is included.

\section{References}

[1] Evolution of Universe to the present inert phase, I.F. Ginzburg, K.A. Kanishev, M. Krawczyk, D. Sokołowska [arXiv:1009.4593] 